\title{
How and Why to Study the Practice Content of a Practice-Based Research Network
}

Kevin A. Pearce, MD, MPH

Margaret M. Love, $P b D$

Mary A. Barron, RN, BSN

Samuel C. Matheny, MD, MPH

Ziyad Mabfoud, PbD

Department of Family Practice and Community Medicine, University of Kentucky, Lexington, Ky

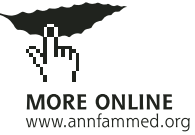

Conflict of interest: none reported

\section{CORRESPONDING AUTHOR}

Kevin A. Pearce, MD, MPH Department of Family Practice and Community Medicine University of Kentucky College of Medicine

Rm K-302, Kentucky Clinic Lexington, KY 40536-0284 kpearce@email.uky.edu

\begin{abstract}
BACKGROUND We describe the rationale, methods, and important lessons learned from doing a practice content study in a new practice-based research network (PBRN).

METHODS We performed a modified replication of the National Ambulatory Medical Care Survey (NAMCS) in the Kentucky Ambulatory Network (KAN). Network clinicians had input into focused modifications of the NAMCS protocol, including addition of data fields of special interest to them. Cross-sectional sampling of patient visits was done for a 1-year period, with each practice collecting data during 2 separate weeks. We used selected results to illustrate lessons learned and the value of this endeavor.
\end{abstract}

RESULTS Twenty-three KAN clinicians helped recruit 33 of their colleagues, and these 56 community-based primary care clinicians collected data on 2,228 office visits. Patient demographics (except race) and the top 10 diagnoses were similar to US NAMCS data. One third of visits addressed 3 or more diagnoses, and one fourth of the visits involved 4 or more medications. The top 10 primary diagnoses represented only one third of all primary diagnoses. Seventy percent of adult patients were either overweight (30\%) or obese (40\%). Rates of counseling on diet or exercise rose with increases in body mass index.

CONCLUSION This study helped us establish and activate our new PBRN, increasing its membership in the process. The descriptive data gained will stimulate, guide, and support our future research activities.

Ann Fam Med 2004;2:425-428. 10.1370/afm.133.

\section{INTRODUCTION}

$\mathrm{P}$ ractice-based research networks (PBRNs) are sustained collaborations between practitioners and academicians dedicated to developing relevant research questions, working together on study design and conduct, and translating new knowledge into practice. ${ }^{1-3}$ Primary care PBRNs have been active in the United States since 1978. Expanded funding opportunities have recently spawned the emergence of many new PBRNs. ${ }^{1,3}$ New PBRNs face the challenges of creating a sense of identity and achieving tangible involvement in research by their members. Selection and conduct of a first study by the network is critical to meeting these challenges. It is advisable to choose a practical and relevant project, avoid complex protocols, and have broad member input in study design and conduct. Methodologic rigor should be balanced against constraints that might seriously limit clinicians' participation. Describing the clinician members and the practice content of a new PBRN can meet these criteria, establish the network's identity, stimulate further research, and enhance the ability of the PBRN to attract funding. The aims of this article are to describe methods for and our experiences with performing a practice content study in a new PBRN. Limited study 
results are included to illustrate important lessons learned and the overall value of this endeavor. Our methods and selected results are available in greater detail as supplemental data in Appendix 1, which can be found online at http://www.annfammed. org/cgi/content/full/2/5/425/DC1).

\section{METHODS}

As we started the Kentucky Ambulatory Network (KAN), we obtained consultation from family physicians with expertise in practice-based research. We thereby perceived the usefulness of doing a practice content study with external comparability, balanced against the risk to member interest of launching the PBRN with a study that did not focus on a clinical question. All our consultants strongly advised that we involve network clinicians when planning any study. We decided to replicate the National Ambulatory Medical Care Survey (NAMCS) within KAN, inviting limited changes to its methods, and involving community-based clinicians in crafting the exact methods. The NAMCS is a survey of visits to office-based physicians in the United States, performed annually by the Centers for Disease Control and Prevention. Family and general practice physicians are combined in reports that are stratified by specialty. ${ }^{4}$ Results of NAMCS replication studies have been published by 2 other primary care PBRNs, ${ }^{5,6}$ with the Ambulatory Sentinel Practice Network (ASPN) publishing the only such comprehensive report a decade ago. ${ }^{5}$

Much of our inaugural convocation of members (November 2000) was devoted to planning the first KAN study. During breakout sessions KAN members and our consultants discussed ideas for modifications to the NAMCS methods. A steering committee was composed of physicians in private practice and faculty from the 2 Kentucky medical schools. Guided by input from the general membership, this committee adopted focused modifications of the NAMCS methods. The KAN General Advisory Committee and the University of Kentucky (UK) Institutional Review Board (IRB) reviewed and approved the protocol. Participating practitioners were deemed the study subjects, because they were recording their impressions about patient visits after the visit was completed. All recorded patientlevel data were free of identifiers, and our IRB agreed that patient consent was not required.

We used the 1997-1998 NAMCS data collection protocol as our starting point. ${ }^{7}$ The steering committee added the following data fields to the patient record data form (Appendix 1, Figure 1): tobacco use, weight and height measurements, and whether chronic pain, depression, or anxiety (each defined by the clinician) contributed to the visit. The numbers of patient complaints, diagnoses, and medications that could be recorded were expanded.

We invited all community-based primary care clinicians (physicians and their affiliated nurse-practitioners and physician assistants) enrolled as KAN members by December 1, 2001, to participate. One of 3 faculty members from the UK Department of Family Practice called at least 1 clinician at each of 40 practices to discuss the study and invite their participation. Subsequently, the KAN study coordinator arranged a site visit at each practice that was attended by a UK faculty member and the study coordinator, with lunch provided by KAN. During this visit, we shared visions for KAN with physicians and staff members, described the purpose and procedures for this first study, and delivered study materials. We identified a clinician leader at each practice site to invite his or her colleagues to the lunch meeting and encourage them to participate.

Each participant completed a brief clinician profile. Participant characteristics were compared with those from a national database of physicians. ${ }^{8}$ Each practice site was provided a study manual and patient record (visit) data forms with serial numbers that facilitated tracking. A single staff person at each practice site was identified as the site coordinator. Participants were instructed to complete the patient record form immediately after each visit or by end of the day. The KAN coordinator proactively contacted the practices throughout the data collection period and encouraged the participants to contact her as needed.

Data were collected from May 2001 to June 2002. Each participating practice volunteered for 2 separate weeks in the calendar year during which the clinicians were expected to complete 25 ambulatory care patient records per participating clinician per week. Clinicians were instructed to spread data collection evenly and systematically throughout each week, recording a set ratio of visits toward the target number (eg, a physician who regularly saw 125 patients per week would fill out a patient record form after every fifth visit). Missing data were not imputed. Data management and analytic methods, including those related to clustering, are described as supplemental data in Appendix 1.

\section{RESULTS}

Fifty-six clinicians at 24 sites returned 2,228 patient record (visit) forms. Forty-six clinicians completed 2 weeks of data collection, the rest completed only 1 week. Initially, 23 clinicians agreed to participate. They helped involve 33 more of their colleagues in the study. Some clinicians completed fewer than 25 patient record forms per week because they overestimated the number of patients they would see that week. The distribution 
of patient record data among clinicians and practices is displayed in Appendix 1. Sixty eight percent of eligible KAN clinicians participated. Available data describing participating and nonparticipating KAN clinicians, plus comparisons with the American Academy of Family Physicians database, are displayed in Appendix 1, Table 1.

\section{Selected Practice Content Findings and Comparisons}

The following brief summaries of a few of our findings illustrate the usefulness of such data in terms of (1) formulating hypotheses, guiding study selection, attracting collaborators, and competing for funding; and (2) the positive impacts of clinician input on study design. Wherever possible, to provide context for their interpretation, we compared our findings with the NAMCS data set, ${ }^{9}$ the data set from the ASPN replication of NAMCS, ${ }^{5}$ and other pertinent databases. More details about these selected findings appear in the online appendix.

Patient visit demographics constitute a treasure-trove of information that a PBRN can use to guide its research endeavors and to provide evidence for comparableness (or uniqueness) of its patients and its study results. The age distribution of patient visits to KAN practices was skewed slightly toward younger patients compared with that of NAMCS, and the KAN preponderance $(61 \%)$ of visits from female patients was similar to that observed in NAMCS, ASPN, and other studies of primary care practice content. ${ }^{5,9,10-12}$ Although African Americans made up only $4 \%$ of KAN visits, this figure reflected 2000 US census data for our participants' locations.

Knowing the most common diagnoses made in a PBRN is highly useful for planning research projects. The top 10 primary diagnoses recorded by KAN clinicians were essential hypertension, acute upper respiratory tract infection, diabetes mellitus, acute sinusitis, acute pharyngitis, normal pregnancy, routine infant and child checkup, low back pain, acute bronchitis, and otitis media. These diagnoses accounted for only $34 \%$ of all primary diagnoses. Seven of the KAN top 10 diagnoses were congruent with the top 10 diagnoses in NAMCS and in ASPN. 5,9

Heeding the advice of our participants to modify NAMCS methods to collect data of special interest to them served our collaborative model of research and provided some unique insights into KAN practice content. A few illustrative examples are summarized here, with details available online in Appendix 1.

Expanding the number of diagnoses and medications that could be recorded (NAMCS is limited to 3 diagnoses and 6 medications) improved our ability to describe the complexity of KAN practices. One third of visits addressed 3 or more diagnoses, and 4 or more medications were prescribed or continued at one fourth of all visits.
Adding height and weight measurements provided our members with data on the scope of weight problems among patients seen in our network. Seventy percent of adult visits were made by overweight (30\%) or obese (40\%) patients. This rate of obesity exceeds estimates based on state ${ }^{13}$ and national population-based data. ${ }^{14}$

KAN clinicians wanted to know whether diet or exercise counseling were associated with body mass index. Combined frequencies of counseling or referral for diet or exercise were significantly higher for obese patients $(49 \%)$ than for overweight $(38 \%)$ or normalweight $(37 \%)$ patients $(P=.002$, adjusted for clustering of these data within practices) ${ }^{15}$

\section{DISCUSSION}

The KAN practice content study helped us establish and activate our new PBRN fairly rapidly, fueling growth of our active membership in the process. We believe that clinicians' involvement in planning, our attention to keeping the protocol as user-friendly as possible, and the personalized attention given each participating practice by our study coordinator, each contributed to this project's success. The data gained will inform, guide, and support our research activities for years. We are sharing this information with our members, potential collaborators, funding agencies, and other networks. By showing that we can describe our network at this level of detail, we have attracted other PBRNs as collaborators and are using our data to inform pilot projects and support grant applications.

Our findings are also being used to validate our members' impressions about some of the problems members face and energize them to help design or participate in future studies. For example, the high prevalence of obesity and the rates of counseling about diet and exercise stratified by body mass index have sparked ideas for studying incentives and barriers to promoting healthy lifestyles.

Replicating established methods facilitates comparisons of KAN practice content with other data sets. It will help consumers of our findings discern similarities and differences of importance to them and make judgments about the applicability of these and future KAN study results to populations of interest. Some of our changes to NAMCS methods also yielded data that may be valuable to other researchers. For example, our findings on the types and numbers of diagnoses made and medications prescribed may be useful for further research into the complexity of primary care. Seminal studies into this critical area have been published, but much is left to do. ${ }^{12,16-18}$

This cross-sectional study provided only a snapshot of the practice content of our PBRN. Practice content 
may change with time, but broad similarities between our results and those of similar studies done years apart suggests some stability in the content of general and family practice. Our data reflect only the first 24 community-based practices to become active in KAN. We could not continue this data collection indefinitely because of insufficient funding and manpower. Clinician membership in $\mathrm{KAN}$ is open to all primary care physicians and their affiliated physician extenders in Kentucky (founding members include a few clinicians in neighboring Tennessee), but active KAN members do not represent a random sample of any population of primary care providers.

Because any individual practice collected data for no more than 2 separate weeks, questions requiring longitudinal data cannot be addressed, and individual practices cannot be described. Efficient systems for continuous collection of practice content data in PBRNs would circumvent these limitations and enhance collaborations among PBRNs, thereby empowering them to answer important questions collectively with high degrees of validity and generalizability.

\section{To read or post commentaries in response to this article, see it} online at http://www.annfammed.org/cgi/content/full/2/5/425.

Key words: Research design; practice-based research; delivery of health care; content of care; physicians practice patterns; family practice

Submitted July 25, 2003; submitted, revised, February 2, 2004; accepted February 17, 2004.

Portions of this manuscript were presented at the Third Annual Convocation of the Kentucky Ambulatory Network, December 6, 2002, Lexington, $\mathrm{Ky}$, and at the Convocation of the Federation of Practice Based Research Networks, March 21, 2003, Arlington, Va.

Funding support: This work was supported in part by Grant \# 1 D12 HP 00005 from the Bureau of Health Professions of the US Health Resources and Services Administration (HRSA).

Acknowledgments: The project steering committee comprised James Ballard, MS, Lexington, Ky; Karen Barnes, MD, Lexington, Ky; David Bolt, Vanceburg, Ky; David Greene, MD, Berea, Ky; Richard Haddy, MD, Louisville, Ky; William Pratt, MD, London, Ky; R. Lamont Wood, MD, Madisonville, Ky. The following consultants provided valuable advice as we formed the Kentucky Ambulatory Network and planned its first study: John Beasley, MD; Larry A. Green, MD; Kurt C. Stange, MD, PhD; and Michael Hartsell, MD.

Study participants: The following KAN practitioners collected data for this study: Aliya Ahmed, MD, Vanceburg, Ky; John Asriel, MD, Glasgow, Ky; Fadi Bacha, MD, Lexington, Ky; Leon Bargo, ARNP, Barbourville, Ky; John Bennett, MD, Georgetown, Ky; Scott Black, MD, Barbourville, Ky; Mary Branstetter, ARNP, Glasgow, Ky; Kelly Burgess, MD, Georgetown, Ky; Craig Burrows, MD, Frenchburg, Ky; Gus Bynum, MD, Georgetown, Ky; Amy Canary, PA-C, Vanceburg, Ky; Baretta Casey, MD, Pikeville, Ky; Jill Crawford, ARNP, Georgetown, Ky; John Darnell, MD, Ashland, Ky; Michael Dunaway, MD, Flemingsburg, Deborah Edelen, ARNP, Lancaster, Ky; Mark Fairchild, PA-C, Vanceburg, KY; Larry Fields, MD, Ashland, Ky; Josephine Glaser, MD, Lexington, Ky; Carmela Fletcher-Green, PA-C, Morehead, Ky; David Greene, MD, Berea, Ky; L. Daniel Hall, MD, Ashland, Ky; Mike
Hamilton, MD, Mt. Vernon, Ky; Mike Hartsell, MD, Greeneville, Tenn; Don Hughes, ARNP, Frenchburg, Ky; Narenda James, MD, Stanford, Ky; Sylvia Kerr, CNM, Morehead, Ky; RaeJean Wallingford McDonald, Flemingsburg, KY; Sandra McGrath, ARNP, Union City, Tenn; William Melahn, MD, Morehead, Ky; James Miller, MD, Stanford, KY; Katherine Miller, MD, Lexington, Ky; Brett Oliver, MD, Georgetown, Ky; Paul C. Pedersen, MD, Barbourville, Ky; William Pratt, MD, London, Ky; H.R. Preston, MD, Georgetown, Ky; Colin Raitiere, MD, Lancaster, Ky; Christopher Sims, MD, Stanford, Ky; Chris Smalley, MD, Frenchburg, Ky; Julie Taylor, MD, Lexington, Ky; Robert Thomas, MD, Ashland, KY; Gwen Webb, RN, COGNP, Vanceburg, Ky; Raymond Wechman, MS, MD, Georgetown, Ky; Randall Wilson, MD, Bryantsville, Ky; Glenn Womack, MD, Flemingsburg, Ky.

\section{References}

1. Nutting PA, Beasley JW, Werner JJ. Practice-based research networks answer primary care questions. JAMA. 1999;281:686-688.

2. Thomas Paul, Griffiths F, Kai J, O'Dwyer A. Networks for research in primary health care. BJM. 2001;322:588-590.

3. Agency for Healthcare Research and Quality. Fact Sheet: Primary care practice-based research networks. Available at: http://www.ahrq. gov/research/pbrnfact.htm. Accessed January 2004.

4. National Ambulatory Medical Care Survey Home Page. National Center for Health Statistics. Centers for Disease Control. Available at: http://www.cdc.gov/namcs. Accessed January 2004.

5. Green LA, Miller RS, Reed FM, Iverson DC, Barley GE. How representative of typical practice are practice-based research networks? Arch Fam Med. 1993;2:939-949.

6. Bennett P, Main DS, Holcomb S, Pace W. Tobacco cessation counseling among underserved patients: a report from CaReNet. J Fam Pract. 2002;51:65-69.

7. US Department of Health and Human Services. Ambulatory Care Visits to Physician Offices, Hospital Outpatient Departments, and Emergency Departments: United States 1997. Vital and Health Statistics 1999; Series 13: No 143.

8. American Academy of Family Physicians Web site: Facts about Family Practice. Available at: http://www.aafp.org/x530.xml. Accessed January 2004 .

9. US Department of Health and Human Services. National Ambulatory Medical Care Survey: 1995-96 Summary. Vital Health Stat. 1999; Series 13: No 142.

10. Marsland DW, Wood MB, Mayo F. A data bank for patient care, curriculum, and research in family practice: 526,196 patient problems. J Fam Pract. 1976;1:25-28.

11. Rosenblatt RA, Cherkin DC, Schneeweiss R, et al. The structure and content of family practice: current status and future trends. J Fam Pract. 1982;4:681-672.

12. Stange KC, Zyzanski SJ, Jaen CR, et al. Illuminating the "black box": a description of 4454 patient visits to 138 family physicians. J Fam Pract. 1998;46:377-389.

13. Behavioral Risk Factor Surveillance System Home Page. Centers for Disease Control. 2003. Available at: http://apps.nccd.gov/brfss/index. asp. Accessed June 2003.

14. National Health and Nutrition Examination Survey Home Page. Centers for Disease Control. 2003. Available at: www.cdc.gov/nchs/ nhanes.htm. Accessed June 2003.

15. Rao JNK, Scott AJ. On simple adjustments to chi-square tests with sample survey data. Ann Stat. 1987; 15:385-397.

16. Flocke SA, Frank SH, Wenger DA. Addressing multiple problems in the family practice office visit. J Fam Pract. 2001;50:211-216.

17. Braddock III CH, Edwards KA, Hasenberg NM, Laidley TL, Levinson W. Informed decision making in outpatient practice: time to get back to basics. JAMA. 1999;282:2313-2320.

18. Rost K, Nutting P, Smith J, et al. The role of competing demands in the treatment provided primary care patients with major depression. Arch Fam Med. 2000;9:150-159. 